\title{
Implementação de um programa de mentoring para estudantes de graduação em saúde: a experiência da FMRP-USP
}

\author{
Implementation of a mentoring program for undergraduate health students: the experience of FMRP-USP \\ Gisele Curi de Barros ${ }^{1}$ (D) $\mid$ giselecaep@fmrp.usp.br \\ Victor Evangelista de Faria Ferraz' (D) vferraz@fmrp.usp.br \\ Maria Paula Panúncio-Pinto' ${ }^{1} \mid$ mapaula@fmrp.usp.br
}

\section{RESUMO}

Introdução: Discussões acerca da importância de proporcionar uma formação integral a estudantes do ensino superior têm sido ampliadas. Para além da aquisição de competências técnicas, ressalta-se a importância do desenvolvimento relacional, de atitudes e valores sustentados em princípios éticos. No campo da formação em saúde, cuidar dos futuros cuidadores pode ser uma forma de atingir tais objetivos. Programas de mentoria configuram uma relação de ajuda em que uma pessoa mais experiente na área de formação acolhe, orienta e auxilia um grupo de ingressantes na mesma área, contribuindo para o desenvolvimento acadêmico, pessoal e social.

Relato de experiência: Este trabalho relata a implementação, o desenvolvimento e a manutenção de um programa de mentoring para cursos da área da saúde em uma instituição pública de ensino superior, sob coordenação do Centro de Apoio Educacional e Psicológico. Foi iniciado por meio de um projeto-piloto com o curso de Medicina e oferecido posteriormente aos demais cursos da unidade de ensino. Caracteriza-se como atividade extracurricular, de caráter voluntário, oferecida no primeiro semestre aos estudantes ingressantes. Os encontros são conduzidos por mentores, auxiliados por estudantes veteranos, os peers.

Discussão: São destacados fatores que contribuíram para maior ou menor sensibilização e compreensão dos objetivos do programa, adesão dos participantes e manutenção dos grupos. O programa recebeu ajustes e modificações alinhados às próprias transformações ocorridas no centro responsável por sua coordenação, com o investimento em atividades coletivas de promoção de bem-estar e qualidade de vida. Conclusão: Programas de mentoria são intervenções importantes para acolhimento e suporte a estudantes durante a formação universitária. Podem apresentar desafios em sua implementação e manutenção, necessitando de coordenação e acompanhamentos constantes, em diálogo com todos os envolvidos em sua realização.

Palavras-chave: Acolhimento; Educação Superior; Estudantes; Mentores; Saúde.

\begin{abstract}
Introduction: Discussions on the importance of providing comprehensive education to students in higher education have been broadened. In addition to the acquisition of technical skills, the importance of relational development, attitudes and values based on ethical principles is emphasized. In the field of health education, taking care of future caregivers can be a way to achieve these goals. Mentoring programs constitute a help relationship in which a person with more experience in the field of training welcomes, guides and assists a group of newcomers in the same area, contributing to academic, personal and social development.
\end{abstract}

Experience report: This work reports on the implementation, development and maintenance of a Mentoring Program for students from undergraduate health courses of a public higher education institution, under the coordination of its educational and psychological support center. It was initiated through a pilot project with medical students, and later offered to students from six other undergraduate health courses of the teaching unit. It is characterized as a voluntary, extracurricular activity, offered in the first semester to incoming students. The meetings are conducted by mentors, assisted by veteran students, the peers.

Discussion: The study highlights factors that contributed to a greater or lesser awareness and understanding of the program's objectives, participation and the maintenance of groups. The program was adjusted and modified in line with the changes that took place at the center responsible for its coordination, with investment in collective activities to promote well-being and quality of life.

Conclusion: Mentoring programs are important interventions for welcoming and supporting students during their undergraduate training. They can present challenges in their implementation and maintenance, requiring coordination and constant monitoring, in dialogue with all those involved in their implementation.

Keywords: Reception; Higher Education; Students; Mentors; Health.

${ }^{1}$ Faculdade de Medicina de Ribeirão Preto, Ribeirão Preto, São Paulo, Brasil.

Editora: Patrícia Lacerda Bellodi.

Recebido em 03/03/21; Aceito em 01/04/21.

Avaliado pelo processo de double blind review. 


\section{INTRODUÇÃO}

As instituições de ensino superior têm tido a missão de proporcionar uma educação integral aos seus estudantes, para além de conhecimentos técnicos ${ }^{1}$. A formação universitária na área da saúde constitui campo para aquisição de habilidades e competências necessárias e condizentes com a atuação profissional, e também vem sendo compreendida como espaço para desenvolvimento de competências relacionais, atitudes e valores ancorados em princípios éticos, o que inclui atenção às necessidades emocionais de seus estudantes, que serão eles mesmos futuros cuidadores ${ }^{2}$.

O processo de formação acadêmica pode ser atravessado por dificuldades e desafios em vários momentos, tanto de ordem pessoal como relacionados aos contextos de ensinoaprendizagem $^{3-6}$. Quando necessidades importantes dos estudantes têm potencial de impactar seu desempenho, sua sensação de pertencimento, seu projeto de vida e desenvolvimento profissional, reconhecê-las, compreendê-las e avaliá-las pode levar à criação e ao estabelecimento de redes de suporte ${ }^{7-10}$.

Os programas de mentoria, ou mentoring, se inserem em um tipo de cuidado global destinado ao estudante, com foco tanto em necessidades acadêmicas e profissionais quanto de ordem pessoal e emocional.

A mentoria é uma relação especial em que alguém com experiência em determinada área de formação profissional acolhe, ajuda e orienta uma pessoa iniciante, contribuindo para seu desenvolvimento integral ${ }^{11}$.

A mentoria se diferencia de outras ações de apoio ao estudante, como a preceptoria, a tutoria acadêmica e a supervisão, as quais se relacionam ao desenvolvimento de competências e à aprendizagem, com a mediação de um preceptor ou um tutor. A mentoria, por sua vez, pressupõe um conhecimento do mentor sobre as características do processo de ensino-aprendizagem, mas não está centrada no processo de aquisição e desenvolvimento de habilidades específicas ${ }^{12}$.

Os programas de mentoria têm se estabelecido em diferentes áreas, como engenharia, administração de empresa e no campo da saúde ${ }^{13,14}$. Para este último, especialmente as escolas de ensino médico preconizam a utilização dessa estratégia, visando à oferta de suporte e acolhimento ao longo de todo o percurso na graduação, bem como em momentos críticos da formação, amplamente documentados pela literatura ${ }^{15,16}$.

Os programas de mentoring assumem diferentes características, podendo ser de caráter individual (par a par) ou grupal, mentoria de pares, ao longo de toda a formação ou em momentos específicos. De maneira geral, a literatura destaca o modelo que envolve um mentor e um estudante como o mais comum no contexto da educação médica ${ }^{17,18}$.

Este trabalho relata a experiência da Faculdade de
Medicina de Ribeirão Preto da Universidade de São Paulo (FMRPUSP) na implementação e no desenvolvimento do Programa de Mentoring para estudantes de seus cursos de graduação na área da saúde, sob coordenação do Centro de Apoio Educacional e Psicológico (CAEP). Discute os desafios para sua manutenção, bem como as perspectivas futuras, levando em conta as características da instituição, os fatores que contribuem para sua continuidade e os aspectos que necessitam de revisão e aprimoramento.

\section{RELATO DE EXPERIÊNCIA}

\section{O Centro de Apoio Educacional e Psicológico da FMRP-USP}

A FMRP-USP tem 69 anos de existência e há 30 conta com um serviço de assistência a seus estudantes, o CAEP.

Inaugurado em 1990, o serviço foi criado em decorrência de demandas apresentadas pela Comissão de Graduação (CG), relacionadas à preocupação com a saúde mental dos estudantes do curso de Medicina, e desde então está vinculado a essa comissão. As discussões iniciais para implantação do serviço ganharam consistência quando se reconheceu a necessidade de cuidado e atenção destinados aos estudantes, considerando as dificuldades emocionais e acadêmicas que eles experimentam ao longo da trajetória universitária. A implantação do serviço consideraria a integralidade da formação acadêmica dos estudantes, que envolve aspectos psicológicos, psicoeducacionais e contextuais. O CAEP foi proposto para atender ao estudante e às suas eventuais dificuldades, e também para prover apoio institucional ao ensino e aos professores ${ }^{19}$.

O CAEP tem coordenação exercida por professores da instituição e conta com um conselho consultor, formado também por docentes indicados pela CG. A equipe técnica, que inicialmente contava com uma psicóloga e uma pedagoga ${ }^{19}$, atualmente é formada por psicopedagoga, pedagogo e duas psicólogas, e por uma servidora que desempenha funções técnico-administrativas ${ }^{20}$.

No período de criação do CAEP, a FMRP-USP contava com o curso de Medicina e com o curso de Ciências Biológicas - Modalidade Médica. As diretrizes que subsidiaram sua estruturação relacionavam-se com aspectos da formação médica em geral. A partir de 2002, o serviço passou a atender às demandas de outros cursos da área da saúde que foram sendo implantados na unidade. Atualmente a FMRP conta com sete cursos de graduação: Medicina, Fisioterapia, Fonoaudiologia, Nutrição e Metabolismo, Terapia Ocupacional, Informática Biomédica e Ciências Biomédicas.

As atividades do centro baseiam-se nos pilares do ensino, com assessorias à CG e aos docentes da FMRP; da pesquisa, com o desenvolvimento de projetos e pesquisa em educação 
e formação em saúde, além de ações de caracterização pedagógica, psicossocial e psicopedagógica de sua população estudantil; e da assistência, com o desenvolvimento de atividades de suporte psicopedagógico e psicológico individual aos estudantes, bem como intervenções de âmbito coletivo, como oficinas temáticas, grupos reflexivos, rodas de conversa e o desenvolvimento de programas como a tutoria acadêmica e o Programa de Mentoring ${ }^{20}$.

\section{A implementação de um programa de mentoria na FMRP-USP}

Quando se criou o programa de mentoria na instituição, ele foi batizado de "Programa de Tutoria Mentoring" e, ao longo dos anos, passou a ser tratado coloquialmente de tutoria. Igualmente os professores e profissionais que atuaram como mentores foram denominados tutores. Apenas recentemente, a partir de 2019, com a oficialização pelo CAEP de um programa de tutoria acadêmica, voltado para estudantes com necessidades educacionais especiais e visando dirimir confusões entre os objetivos de ambos, o Programa de Tutoria Mentoring passou a ser denominado "Programa Mentoring" ou "Programa de Mentoria", e os professores e profissionais envolvidos passaram a ser referidos como mentores. Neste manuscrito, o programa será apresentado com o nome "Programa de Tutoria Mentoring" apenas com a finalidade de registro histórico, quando do momento de sua criação. Posteriormente, será tratado como Programa de Mentoria, para evitar as confusões já elucidadas.

As reflexões preliminares sobre uma proposta de apoio aos estudantes na USP ocorreram por meio da designação de um grupo de trabalho nomeado pela Pró-Reitoria de Graduação (PRG). Foi organizado o"I Seminário Pró-Tutorias" em novembro de 1998, para fomentar a discussão sobre as diretrizes para a implantação de Programas de Apoio ao Estudante nos diversos cursos da USP. As unidades de ensino teriam a liberdade para propor suas ações de apoio aos estudantes, de acordo com cada realidade.

A partir dessa iniciativa da PRG-USP, em 2002 foi finalmente implantado, sob gestão do CAEP, o Programa de Tutoria Mentoring na FMRP, e teve início o seu programa-piloto ${ }^{21 .}$

A proposta-piloto apresentada à CG e à direção da unidade retomava as discussões promovidas pela PRG sobre a necessidade de programas de apoio aos estudantes. Além disso, apresentava referências teóricas internacionais sobre programas de mentoria e trazia a experiência de implantação de programas similares no contexto brasileiro - como o Programa da Faculdade de Medicina da USP (FMUSP) ${ }^{15}$, que poderia servir de inspiração para a atividade na FMRP. Por fim, o documento trazia a proposição de "piloto" a ser realizado no curso de Medicina, com os estudantes do primeiro semestre.

O programa se propôs a contribuir para a construção da identidade profissional, facilitar a adaptação à universidade, identificar, acolher e prevenir eventuais dificuldades emocionais, e potencializar o desenvolvimento das relações entre os estudantes e entre as categorias discente e docente dentro da unidade. Após sua aprovação, o Programa de Mentoria da FMRP passou a ser operacionalizado em 2003, com base nas seguintes etapas:

- Divulgação do programa com os esclarecimentos sobre a proposta de mentoria e convite formal a docentes da FMRP e médicos contratados do Hospital das Clínicas da FMRP-USP para adesão ao programa (perfil adequado à atividade e carga horária compatível com as necessidades do programa).

- Capacitação pela equipe técnica do CAEP para instrumentalizar os inscritos em relação à natureza, ao objetivo e à operacionalização da atividade destinada aos ingressantes no curso de Medicina em 2003.

- Divulgação do programa com os esclarecimentos sobre a proposta de mentoria e convite formal a estudantes de outros anos do curso de Medicina (veteranos), para que pudessem colaborar com os mentores.

- Divulgação do programa aos estudantes ingressantes (boletins informativos, ida à sala de aula) e convite para a atividade.

- Inscrição dos estudantes interessados (tanto ingressantes quanto veteranos) feita na Seção de Alunos da faculdade.

- Organização dos grupos de mentoria contando com uma dupla de mentores em cada grupo, um do ciclo básico e um do clínico (sugestão do CAEP).

- Início do programa no segundo semestre de 2003, com a proposição de reuniões quinzenais, em horário de almoço (preferência dos estudantes), com duração máxima de uma hora e meia.

- Assessoria aos mentores pela equipe técnica do CAEP, em parceria com seu grupo de consultores, por meio de reuniões mensais, com os seguintes objetivos: acompanhar e avaliar as atividades desenvolvidas, aperfeiçoar o programa, discutir eventuais dificuldades e elaborar estratégias futuras para a incorporação, quando possível e pertinente, de outros grupos de mentorandos. 
Formaram-se dez grupos, com uma média de seis estudantes do primeiro ano (ingressantes) e dois estudantes veteranos em cada grupo, além do mentor. $O$ piloto foi bem avaliado pelos participantes, o que validou a continuidade da atividade e o estabelecimento de um programa de mentoria na FMRP. Por meio de uma articulação com a CG, foi possível inserir a primeira reunião dos grupos de mentoria no calendário de atividades da "Semana de Recepção aos Calouros". Essa organização ficou sob responsabilidade do CAEP, cuja equipe organizava os grupos previamente por meio de acesso, na Seção de Alunos, à lista de aprovados no vestibular.

À medida que o programa se consolidou no curso de Medicina, foi apresentado aos demais cursos criados na unidade a partir de 2002. Em 2006, houve a inserção do curso de Nutrição; em 2007, dos cursos de Fisioterapia e Fonoaudiologia; e, em 2008, do curso de Terapia Ocupacional. Em 2009, o programa também se estendeu ao curso de Informática Biomédica. $\mathrm{O}$ CAEP passou a realizar um evento de encerramento ao final do primeiro semestre, reunindo todos os envolvidos na mentoria (mentores, veteranos peers e estudantes ingressantes) para troca de experiências e avaliação da atividade.

No período de 2009 a 2016, o programa manteve sua estrutura como atividade extracurricular/voluntária para seis de seus cursos, com ajustes pontuais buscando maior adesão de estudantes, mentores e peers.

O curso de Ciências Biomédicas foi o último a aderir ao Programa de Mentoria da FMRP e, por tratar-se do curso mais novo da unidade, contou desde sua implantação com tutoria acadêmica como atividade regular, uma disciplina da matriz curricular, ao longo de todo o curso. Contudo, em 2016 o curso aderiu ao Programa de Mentoria para estudantes ingressantes e, em seu primeiro semestre, ofereceu sua tutoria curricular nos moldes e objetivos de mentoria, sob condução e responsabilidade do CAEP.

\section{O Fórum de Planejamento Estratégico do CAEP e as repercussões no Programa de Mentoria}

Em julho de 2016, foi realizado o Fórum de Planejamento Estratégico do CAEP, atendendo a uma necessidade de reestruturação do serviço. $A$ atividade contou com a participação de várias instâncias da faculdade (direção, docentes, coordenadores de cursos, estudantes e funcionários) e estabeleceu quatro eixos de discussão: assistência psicológica e psicopedagógica aos estudantes; apoio ao ensino; desenvolvimento docente; e pesquisa em educação superior em saúde. No primeiro eixo, foi destacado o investimento em estratégias de prevenção de doenças e promoção de saúde e qualidade de vida.

A mentoria não se caracteriza como atividade de cuidado específico em saúde mental. Entretanto, considerando a preconização de programas voltados ao cuidado integral destinado aos estudantes, ela pode ser muito útil ao englobar ações preventivas e de apoio. Os espaços de discussão nos grupos de mentoria proporcionam acesso e troca de informações, detecção precoce de necessidades (financeiras, acadêmicas, psicossociais) e possíveis direcionamentos a outras instâncias e órgãos da universidade, de acordo com as demandas dos estudantes.

Desse modo, o CAEP se propôs a investir na ampliação do programa, contribuindo para a reorientação das prioridades no serviço: maior envolvimento da comunidade acadêmica (docentes, estudantes e coordenação de cursos) em ações de cuidado integral, expandindo as estratégias focadas no indivíduo para aquelas de âmbito coletivo. De acordo com as diretrizes traçadas no fórum, alguns ajustes foram propostos no Programa de Mentoria a partir de 2016.

As mudanças ocorreram em várias etapas, desde o planejamento do programa e o envolvimento de todos os cursos da FMRP até a concretização dos encontros de mentoria. Reconheceu-se a importância de alocar mais uma profissional de psicologia para auxiliar na coordenação do programa. As demandas de uma atividade dessa natureza, em uma instituição ampla e diversa, pedem um corpo técnico mais robusto para seu acompanhamento.

Como uma ação geral inicial, em outubro de 2016 foi aberto um edital, divulgado entre todos os docentes da unidade, profissionais contratados do Hospital das Clínicas da FMRP e pós-graduandos. A estratégia permitiu ampliar as informações sobre o programa a toda comunidade, ainda desconhecida por alguns, e possibilitar a inserção de novos integrantes. Ainda no sentido de divulgar e estimular a participação de alunos veteranos de todos os cursos (peers), também se optou por abertura de edital com os mesmos propósitos. A escolha por lançar os editais foi motivada pelo fato de que o programa vinha contando, havia algum tempo, com os "mentores da casa", familiarizados com o CAEP. Embora fosse importante contar com a sua permanência, percebeu-se a necessidade de renovação e ampliação para a participação de novos mentores.

A partir da divulgação dos editais, o CAEP recebeu inscrições para a mentoria em todos os cursos, e o processo de seleção de mentores e estudantes peers envolveu a realização de entrevistas individuais com os candidatos, objetivando escutar a experiência do mentor (no caso de já ter participado), ouvir as opiniões e expectativas dos que não conheciam o programa, além de fornecer informações sobre a atividade. Após a seleção dos mentores e peers, foi ofertada capacitação antes do início do programa, no primeiro semestre de 2017.

Os grupos de mentoria foram realizados entre os 
meses de março e junho de 2017, com frequência semanal ou quinzenal, e o primeiro encontro dos estudantes com os respectivos mentores e peers ocorreu durante a "Semana de Recepção aos Calouros". O CAEP passou a realizar reuniões periódicas e sistemáticas de interlocução sobre o andamento do programa em cada grupo/curso, com compartilhamento de experiências, reflexões e estratégias entre mentores. Também foram realizadas reuniões de interlocução com os peers.

Como atividade prevista do programa, realizou-se o evento de encerramento com todos os grupos. $O$ encontro ocorreu por meio da realização de "grupos de discussão" sobre "aspectos positivos", "aspectos que podem ser aprimorados" e "sugestões para melhorias do programa".

O CAEP passou a adotar essas etapas de divulgação, preparação e operacionalização do programa de forma sistemática, aprimorando a estrutura da atividade.

A Figura 1 apresenta uma síntese dos acontecimentos importantes ligados ao Programa de Mentoria, uma linha do tempo com início na criação do CAEP, passando pela implantação do Programa de Mentoria, pelas principais mudanças efetuadas e pelos marcos em seu desenvolvimento até os dias atuais.

\section{DISCUSSÃO}

O Programa Mentoring, implementado na FMRP sob coordenação do CAEP, alcançou reconhecimento institucional e de seus participantes. Como estratégia de suporte, acolhimento e orientação aos estudantes ingressantes em um novo contexto de vida, a atividade tem contribuído para tornar a apropriação da vida universitária menos sofrida e mais estimulante.

Para os mentores e peers, também tem trazido satisfação, ao perceberem que sua experiência contribui para o acolhimento e para a sensação de pertencimento dos ingressantes, além da formação de vínculos que muitas vezes ultrapassam o período do oferecimento do programa e se mantêm ao longo do curso.

Compreende-se que o programa precisou ser revisto e modificado desde sua implementação. Existem desafios para sua manutenção relacionados ao contexto institucional, à natureza da atividade e à sua coordenação.

Por ser atividade voluntária, depende de inscrições para ter seu oferecimento minimamente garantido. Apesar do desenvolvimento de estratégias de divulgação e adesão, a participação não deve ser "imposta" apenas para cumprir uma obrigação. Porém, programas ganham apoio institucional pela existência de demandas para criação de espaços que ofereçam suporte aos estudantes. Nesse sentido, cria-se um certo paradoxo, em que há demanda por atividades que promovam bem-estar, mas dificuldades em operacionalizá-las.

Para os estudantes, as principais queixas e dificuldades para a adesão relacionam-se à "concorrência" com outras atividades ofertadas (ligas acadêmicas, esportivas e treinos). Aspectos relacionados à natureza da atividade envolvem a não compreensão dos objetivos e das finalidades da mentoria, não identificação com a proposta ou com mentores, o que corrobora experiências já relatadas na literatura ${ }^{22,23}$. A sobrecarga de trabalho e a falta de reconhecimento formal desse tipo de atividade contribuem para um menor envolvimento dos docentes ${ }^{17,18}$.

Da parte do CAEP, a experiência adquirida ao longo dos 18 anos de implementação do programa convida a reflexões. Se os mentores oferecem ajuda por conta de sua experiência adquirida ao longo do tempo, servindo como guia e ofertando caminhos e possibilidades de acolhimento a quem chega, o centro também se responsabiliza por tomar para si tais premissas, na função de coordenação da mentoria.

Figura 1. Evolução do CAEP e do Programa de Mentoria.

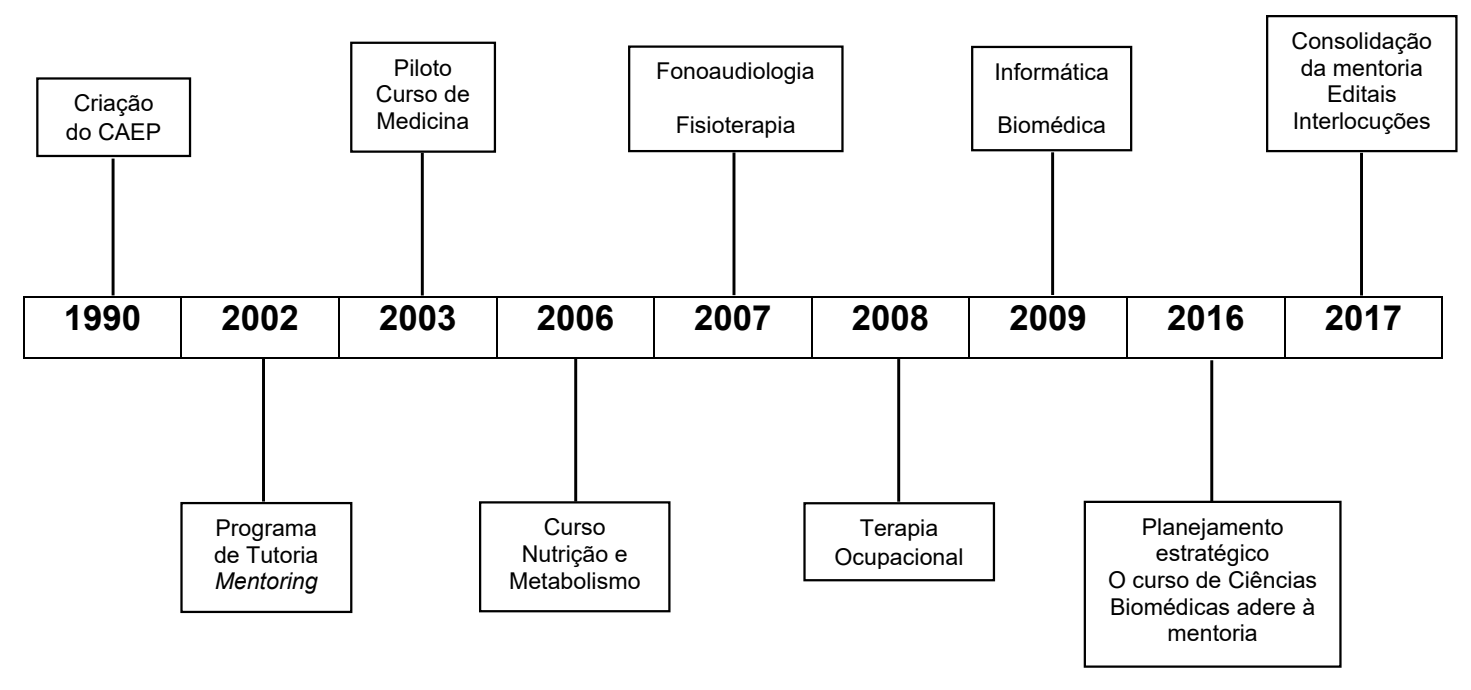


A cada ano, uma nova edição do programa começa, e a função de acolher, guiar, acompanhar, trocar experiências e avaliar é um movimento que se inicia no CAEP com os envolvidos no programa. Mais do que formas prescritivas que sirvam a todos, entende-se que o acompanhamento se faz no varejo, de forma artesanal, considerando as particularidades de cada área, de cada curso, de cada grupo com seu mentor, peer e calouros. A formação integral em saúde e o cuidado destinado a estudantes e mentores representam uma infinita jornada de possibilidades. Mantê-la viva depende de confiar, como Mentor confia nas capacidades de Telêmaco ${ }^{24}$, que um programa é capaz de se manter ao longo do tempo enquanto os encontros que ele proporciona possam promover vínculos férteis com mentores e estudantes, destes entre si e da instituição para com eles.

\section{CONCLUSÃO}

Os programas de mentoring são atividades importantes de suporte, acolhimento e desenvolvimento dos estudantes. O planejamento, o acompanhamento e a avaliação constantes fazem-se necessários para que sejam cumpridos os objetivos e para que a ação possa ser significativa para os que dela participam, com potencial agregador e atrativo.

\section{CONTRIBUIÇÃO DOS AUTORES}

Gisele Curi de Barros participou da escrita integral do relato de experiência e da revisão de todas as partes. Victor Evangelista de Faria Ferraz participou da revisão do relato de experiência. Maria Paula Panúncio-Pinto participou da escrita do resumo em inglês, da revisão de todas as partes e da elaboração da Figura 1.

\section{CONFLITO DE INTERESSES}

Declaramos não haver conflito de interesses.

\section{FINANCIAMENTO}

Declaramos não haver financiamento.

\section{REFERÊNCIAS}

1. Panúncio-Pinto MP, Colares MFA. O estudante universitário: os desafios de uma educação integral. Medicina (Ribeirão Preto). 2015;48(3):273-81.

2. Esperidião $E$, Munari DB. A formação integral dos profissionais de saúde: possibilidades para a humanização da existência. Ciênc Cuid Saúde. 2005;4(2):163-70.

3. Neves MCC, Dalgalarrondo P. Transtornos mentais auto-referidos em estudantes universitários. J Bras Psiquiatr. 2007;56(4):237-44. doi: 10.1590/ S0047-20852007000400001.

4. Aguiar SM, Vieira APGF, Vieira KMF, Aguiar SM, Nóbrega JO. Prevalência de sintomas de estresse nos estudantes de medicina. J Bras Psiquiatr. 2009;58(1):34-8. doi: 10.1590/S0047-20852009000100005.
5. Fiorotti KP, Rossoni RR, Borges LH, Miranda AE. Transtornos mentais comuns entre os estudantes do curso de medicina: prevalência e fatores associados. J Bras Psiquiatr. 2010;59(1):17-23. doi: 10.1590/S004720852010000100003.

6. Padovani RC, Neufeld CB, Maltoni J, Barbosa LNF, Souza WF, Cavalcanti HAF, et al. Vulnerabilidade e bem-estar psicológicos do estudante universitário. Rev Bras Ter Cogn. 2014;10(1):2-10. doi: 10.5935/1808-5687.20140002.

7. Cerchiari EAN, Caetano D, Faccenda O. Utilização do serviço de saúde mental em uma universidade pública. Psicol Ciênc Prof. 2005;25(2):252-65.

8. Pan M, Zonta GA, Tovar A. Plantão institucional: relato de uma intervenção psicológica na UFPR. Psicol Estud. 2016;20(4):555-62 [acesso em 2 mar 2021]. Disponível em: http://www.periodicos.uem.br/ojs/index.php/ PsicolEstud/article/view/27594.

9. Campos CRF, Oliveira MLC, Mello TMVF, Dantas CR. Academic performance of students who underwent psychiatric treatment at the students' mental health service of a Brazilian university. Sao Paulo Med J. 2017;135(1):23-8. doi: 10.1590/1516-3180.2016.017210092016.

10. Malajovich N, Vilanova A, Frederico C, Cavalcanti MT, Velasco LB. A juventude universitária na contemporaneidade: a construção de um serviço de atenção em saúde mental para estudantes. Mental. 2007;11(21):356-77 [acesso em 2 mar 2021]. Disponível em: http://pepsic.bvsalud.org/scielo. php?script=sci_arttext\&pid=S1679-44272017000200005\&lng=pt\&tlng=pt.

11. Bellodi PL. Tutoria. In: Bellodi, PL, Martins, MA. Tutoria: mentoring na formação médica. São Paulo: Casa do Psicólogo; 2005. p. 53-67.

12. Botti SHO, Rego S. Preceptor, supervisor, tutor e mentor: quais são seus papéis? Rev Bras Educ Med. 2008;32(3):363-73.

13. Agholor $D$, de Nalda AL, Bárcena NS. Mentoring future engineers in higher education: a descriptive study using a developed conceptual framework. Production. 2017;27(spe):e20162207. doi: 10.1590/0103-6513.220716.

14. Silva CRE. Orientação profissional, mentoring, coaching e counseling: algumas singularidades e similaridades em práticas. Rev Bras Orientaç Prof. 2010;11(2):299-309 [acesso em 3 mar 2021]. Disponível em: http://pepsic.bvsalud.org/scielo.php?script=sci_arttext\&pid=S1679$33902010000200014 \&$ lng $=$ pt\&tlng=pt.

15. Bellodi PL. O Programa Tutores e a integração dos calouros na FMUSP. Rev Bras Educ Med. 2004;28(3):204-14. doi: 10.1590/1981-5271v28.3-027.

16. Martins AF, Bellodi PL. Mentoring: uma vivência de humanização e desenvolvimento no curso médico. Interface (Botucatu). 2016;20(58):71526. doi: 10.1590/1807-57622015.0432.

17. Kashiwagi DT, Varkey $P$, Cook DA. Mentoring programs for physicians in academic medicine: a systematic review. Acad Med. 2013;88(7):1029-37. doi: 10.1097/ACM.0b013e318294f368.

18. Farkas AH, Allenbaugh J, Bonifacino E, Turner R, Corbelli JA. Mentorship of US Medical Students: a systematic review. J Gen Intern Med. 2019;34(11):2602-9. doi: 10.1007/s11606-019-05256-4.

19. Cianflone ARL, Figueiredo JFC, Colares MFA. O Centro de Apoio Educacional e Psicológico (CAEP) da Faculdade de Medicina de Ribeirão Preto (USP): história e perspectivas. Medicina (Ribeirão Preto). 2002;35:392-6.

20. Murakami K, Barros GC, Peres CM, Flauzino RH, Colares MFA. Atuações de um centro educacional e psicológico junto a estudantes universitários. Rev Bras Orientaç Prof. 2018;19(1):109-19. doi: 1026707/1984-7270/2019v19n1p109.

21. Colares MFA, Peres CM. Tutoria na Faculdade de Medicina de Ribeirão Preto (USP). In: Bellodi, PL, Martins, MA. Tutoria: mentoring na formação médica. São Paulo: Casa do Psicólogo; 2005. p. 176-8.

22. Bellodi, PL, Chebabo, R, Abensur, SI, Martins, MA. Mentoring: ir ou não ir, eis a questão: um estudo qualitativo. Rev Bras Educ Med. 2011;35(2):23745. doi: 10.1590/S0100-55022011000200013.

23. Straus, SE, Johnson, MO, Marquez, C, Feldman, MD. Characteristics of successful and failed mentoring relationships: a qualitative study across two academic health centers. Acad Med. 2013 Jan;88(1):82-9. doi: 10.1097/ ACM.0b013e31827647a0.

24. Homero. Odisséia. Tradução Jaime Bruna. São Paulo: Cultrix; 1993.

This is an Open Access article distributed under the terms of the Creative Commons Attribution License, which permits unrestricted use, distribution, and reproduction in any medium, provided the original work is properly cited. 